\title{
Image processing based on the detection of external defects of fan tower Weld
}

\author{
Ren $\mathrm{Wu}^{1}$ and Yuan $\mathrm{Sun}^{2 *}$ \\ ${ }^{1}$ Electrical Engineering, Shanghai Dian Ji university, Shanghai, Shanghai, 200000, China \\ ${ }^{2}$ Machinery Industry, Shanghai Dian Ji university, Shanghai, Shanghai, 200000, China
}

\begin{abstract}
With the continuous development of wind power generation technology and the continuous increase in the demand for electric energy, the height of the fan tower is more and more demanding. It is very important to detect the weld produced in the welding process of fan tower. In this paper, an algorithm for weld defect detection based on traditional image processing and convolutional neural network is proposed. Firstly, the traditional image processing algorithm is used to gray the weld image collected by industrial camera. Then, the gray image of welding seam is enhanced to improve the visual effect and clear the image, which is convenient for further processing and analysis of the image by computer. Finally, the image is used as the input of the trained convolution neural network to judge whether there are defects outside the weld.
\end{abstract}

\section{Introduction}

The tower structure types of wind turbine mainly include cylinder type, truss type, etc. According to the type of materials can be divided into pure steel type, concrete type. Among them, steel cylindrical tower as the main technology is widely used by wind power enterprises at home and abroad.

The fan tower is the tower pole of the wind turbine, which mainly plays a supporting role. In order to obtain sufficient and stable wind energy for power generation, the fan tower should support the wind turbine at an altitude of 60-100 meters. To some extent, in the same environment, the higher the fan tower, the more wind energy the wind turbine can capture. With the increase of the height of the fan tower, in order to ensure its safety performance, The steel used in the production of wind towers is becoming thicker and thicker. At the same time, the tower drum of the fan not only plays a supporting role, but also can absorb the vibration of the unit. It is very important to ensure the welding quality of the tower drum to meet the standard for the safe and stable operation of the wind turbine.

In huaneng huaining longchi(2.2 MW) wind power tower drum, for example, the project, including 20 tower drum tower drum is a kind of conical welded structure, each tower drum is divided into four segments, including section, upper middle period of lower, middle and lower segment and segment, and each paragraph 8-10 section of single section steel roll round, each section of the connection between the flange connection, top mounted wind turbines ${ }^{[1]}$ The welding seams of the fan tower tube mainly include the vertical seam inside and outside of the single tower tube, the inner and outer ring seam behind the two tower tubes, and the splicing weld of the flange and the drum. At present, large-scale wind turbine welding machine is mainly used in tower production in China. However, the nondestructive testing of tower welding seam is still completely dependent on manual work, detect faults include external and internal defect detection, for external defects, mostly use the magnetic particle testing (MT), penetrant testing (PT) and eddy current testing (ET), these testing methods are complex to operate and heavy workload.

\section{Traditional image processing}

After effective traditional image processing, the image has better defect characteristics and higher recognition, and the image quality of weld will directly affect the accuracy of defect detection. In this paper, the image of the original weld is grayed and enhanced.

\subsection{Image graying}

At present, color space of color pictures includes RGB color space, CMY $(\mathrm{K})$ color space, HSV color space and CIE-XYZ color space. The mainstream color space is RGB three-channel color map. RGB images are stored in the form of three-dimensional matrix. When training the convolutional neural network, the three components of RGB need to be processed, which will increase the time and difficulty of network training. Moreover, the color itself is easy to be interfered by factors such as lighting, which makes it difficult to provide accurate key information. Therefore, it is necessary to grayscale the collected color images to reduce the matrix dimension of the images.

After graying, the color information is lost, but the

e-mail: suny@sdju.edu.cn 
gradient information of the image is still retained. In weld defect detection, in order to identify defects, the most critical factor is image gradient, that is, image edge information. The present methods of image graying include component method, maximum method, average method and weighted average method.

The component method means that the brightness values of the three components in the RGB image are respectively taken as the gray values of the three gray images, and one of the gray values is selected according to the subsequent actual requirements. The maximum value method takes the maximum brightness value of three component brightness in RGB image as the gray value of gray image. The mean value method takes the mean value of the brightness value of three components of RGB image as the gray value of the gray image. The weighted average method is to average the three components with different weights. Normally, human eyes are the most sensitive to green and the least sensitive to blue, so the gray value of the gray image will be obtained according to the threecomponent weighted average of RGB image in the formula 1 .

$$
\begin{gathered}
\operatorname{Gray}(i, j)=0.299 \times R(i, j)+0.578 \times G(i, j)+ \\
0.114 \times B(i, j)
\end{gathered}
$$

Where $\mathrm{I}$ and $\mathrm{J}$ are the position coordinates of a pixel point in the image.[2]

In this paper, the weighted average method is used to grayscale the image of the weld. The original image of the weld and the grayscale image after grayscale are shown in the figure.

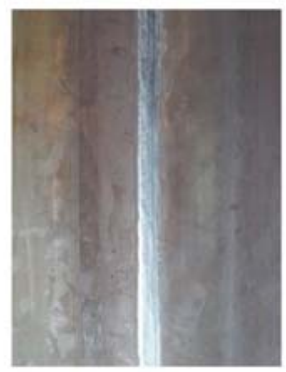

Figure 1. Original drawing of welding seam

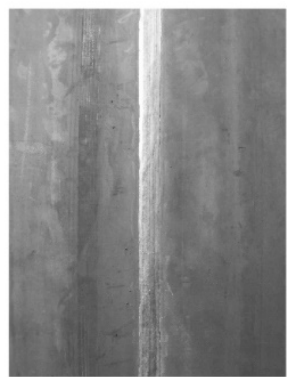

Figure 2. Grayscale image of welding seam

\subsection{Image enhancement}

The main purpose of image enhancement is to improve the quality and recognizability of the image, so that the image is more conducive to observation or further analysis and processing. The image information is mainly conveyed through some features, such as the edge, outline and contrast of the image, etc. By highlighting and enhancing these image features, the effective information of the image can be better displayed and the use value of the image can be improved. Image enhancement can be divided into intra-spatial processing and intra-frequency processing according to the different scopes. Intra-spatial processing is the direct image processing. In the frequency domain, the image transformation coefficient is calculated in a certain transformation domain of the image, and the enhancement effect of the image is obtained by inverse transformation. In this paper, the image sharpening in spatial domain is used to enhance the image of the weld.

Image sharpening mainly refers to edge sharpening, highlighting the edge or texture in the image, so that the various boundaries in the image more obvious. From a mathematical point of view, the image before sharpening is equivalent to the image that has been "integrated", in order to achieve the effect of sharpening is to use the "differential" operation will be "integrated" image restore. In the process of image sharpization, we need to use a variety of edge detection operators, such as Roberts operator, Sobel operator, Prewitt operator and Laplace operator, among which the first three operators are firstorder edge operators and also are differential operators[3]. Laplace operator is usually a second-order edge operator.

For the discrete image $f(x, y)$, the calculation formula of Roberts operator is shown in Figure 2.

$R(i, j)=\sqrt{[f(i, j)-f(i+1, j+1)]^{2}+[f(i, j+1)-f(i+1, j)]^{2}}$

The Roberts operator consists of two second-order templates:

$$
\left[\begin{array}{cc}
1 & 0 \\
0 & -1
\end{array}\right] \quad\left[\begin{array}{cc}
0 & 1 \\
-1 & 0
\end{array}\right]
$$

Sobel operator is two $3 * 3$ matrices, which respectively represent the horizontal gradient and vertical gradient of the image.

$$
\left[\begin{array}{lll}
-1 & 0 & 1 \\
-2 & 0 & 2 \\
-1 & 0 & 1
\end{array}\right] \quad\left[\begin{array}{ccc}
-1 & -2 & -1 \\
0 & 0 & 0 \\
1 & 2 & 1
\end{array}\right]
$$

The size of Prewitt operators is the same as that of Sobel, both of which are $3 * 3$ operators:

$$
\left[\begin{array}{ccc}
-1 & -1 & -1 \\
0 & 0 & 0 \\
1 & 1 & 1
\end{array}\right] \quad\left[\begin{array}{ccc}
-1 & 0 & 1 \\
-1 & 0 & 1 \\
-1 & 0 & 1
\end{array}\right]
$$

The Laplace operator is a second order differential operator independent of the edge direction. It is a scalar rather than a vector. The Laplace operator has the property of rotation invariant, and is often used to extract the edge of an image in image processing. Its expression is as follows:

$$
\nabla^{2} f=\frac{\partial^{2} f}{\partial x^{2}}+\frac{\partial^{2} f}{\partial y^{2}}
$$

The approximate formula of digital image is:

$$
\begin{gathered}
\nabla^{2} f(x, y)=f(x+1, y)+f(x-1, y)+f(x, y+ \\
1)+f(x, y-1)-4 f(x, y)
\end{gathered}
$$

In MATLAB, based on the above four operators, the effect of the weld gray image after sharpening is shown in the figure 3 . 


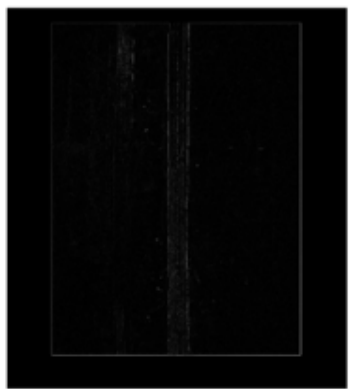

(a) Enhanced image of (b) Enhanced image of the Robert operator

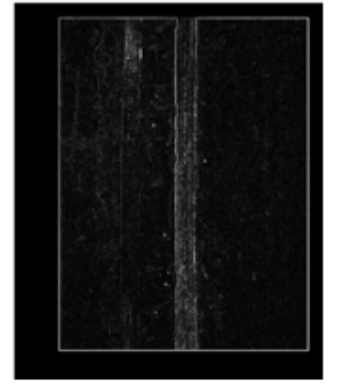

the Laplace operator

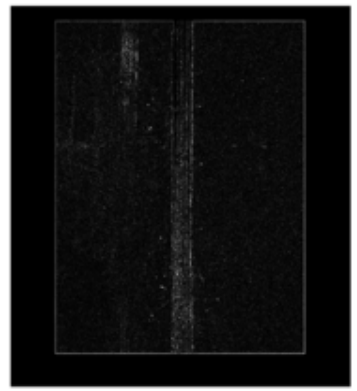

(c) Enhanced image of the Sobel operator

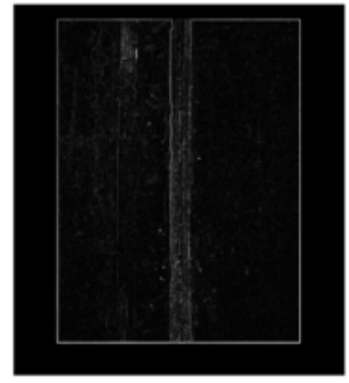

(d) Enhanced image of the Prewitt operator

Figure 3. Enhanced image

It can be seen from FIG. 3 that the sharpened weld is more prominent in the background, and the sharpened image based on Laplace operator has a better sharpening effect than the other three operators. Not only is the edge of the weld accurate and clear, but also the features inside the weld can be effectively reflected. Therefore, the grayscale image of weld is sharpened by Laplace operator.

\section{Structure of convolutional neural network}

Convolutional neural networks (CNNs) are very similar to ordinary neural networks and consist of a large number of neurons. A convolutional neural network is a kind of feed-forward neural network whose neurons can respond to a part of the surrounding units within its coverage. Moreover, the default input of a convolutional neural network is an image. A convolutional neural network consists of many layers whose input and output are threedimensional. A convolutional neural network usually consists of an input layer, a convolutional layer, a pooling layer, a full connection layer and an output layer. Some convolutional neural networks used for classification problems also have a Softmax layer. ${ }^{[4]}$

\subsection{The input layer}

The input layer is the input of the whole neural network. In the convolutional neural network for image processing, the input layer represents the pixel matrix of the input image. A THREEDIMENSIONAL matrix can represent an image, the length and width of the three-dimensional matrix represent the size of the image, and the depth of the three-dimensional matrix represents the color depth of the image. In this paper, the images at the input layer are all grayscale images, so the depth is 1 . Starting from the input layer, the convolutional neural network transforms the three-dimensional matrix of the upper layer into the three-dimensional matrix of the lower layer through different neural network structures until the final full connection layer.

\subsection{Convolution layer}

Convolution layer is the most important part of a convolutional neural network, and the conventional full connection layer, convolution on each node of the input layer is a layer of neural network, a small piece of convolution layer goal is based on neural network in every small piece of in-depth analysis, and thus get higher characteristic, image abstraction degree convolution layer will increase the depth of the original node.

The convolutional layer is composed of many feature mappings, each of which has a convolution kernel. Different feature mappings contain different convolution kernels, and the feature types they can extract are also different. The size of the convolution kernel should be determined according to the size of the image. If the convolution kernel is too large, overfitting will occur. If the convolution kernel is too small, it is difficult to fully extract the effective features of the image.

The size of the convolved image $\mathrm{FW}^{*} \mathrm{FH}$ can be obtained by Formula 5 and 6.

$$
\begin{aligned}
& F W=\frac{(W-F+2 P)}{S}+1 \\
& F H=\frac{(H-F+2 P)}{S}+1
\end{aligned}
$$

Where $\mathrm{W}$ and $\mathrm{H}$ are the width and height of the original image, $\mathrm{F}$ is the size of the convolution kernel, $\mathrm{S}$ is the step size, and $\mathrm{P}$ is the boundary filling size.

The number of parameters to be trained in the convolutional layer is only related to the size of the convolution kernel and the number of the convolution kernel. Meanwhile, all neurons with the same feature map in the convolutional layer have the same convolution kernel, which is called weight sharing. In this way, the number of parameters to be trained and the difficulty of training can be reduced. 


\subsection{Pooling layer}

The function of pooling layer is mainly to reduce the dimension of data on the basis of retaining the useful data of convolutional layer, reduce the computation amount of neural network, and improve the training speed. In other words, pooling layer neural network does not reduce the depth of the matrix, but it can reduce the size of the matrix. After pooling, images with higher resolution can be changed to images with lower resolution, and the number of nodes in the final full connection layer can be further reduced. Pooling also prevents overfitting.

\subsection{The connection layer}

After many times of convolutional layer and pooling layer processing, the input image information has been abstracted into features with higher information content. As the number of convolutional layers and pooling layers increases, the number of feature maps is also increasing, and the number of neurons in each feature map is also decreasing until the number of neurons decreases to 1 . When there is only one neuron in each characteristic plane, the network becomes fully connected. Each node of the full connection layer is connected to all nodes of the previous layer, and the extracted features are summarized.

\subsection{Output layer}

The number of neurons in the output layer is related to the specific task of the network. For weld recognition in this paper, the number of output layers is the type of weld that needs to be detected.

\section{Training and experiment of convolutional neural network}

In this paper, a seven-layer convolutional neural network structure is adopted, including a three-layer convolutional layer, a three-layer pooling layer and a full-connection layer. This paper is based on the 300 pictures of welding seams collected in the tower drum factory. Because the single picture collected is too large and the single picture contains both the weld defect position and the normal weld position, the 300 pictures are expanded to 500 pictures of normal weld through image segmentation, 200 pictures of poor weld forming and weld crack respectively. Due to the limitation of conditions, the number of sample images collected at the present stage is small, which will affect the training effect of the network. In this paper, the defect image, add noise and transformation methods of the location, put the pictures of the normal population expanded to 1200 , of which 1000 zhang used in network training, 200 for network test, and put the pictures of the poor appearance of weld and weld crack images were expanded to 600 pieces, each of 500 pieces used in network training, 100 as a test set for network testing. The pictures of the three training samples are shown in Figure 4.

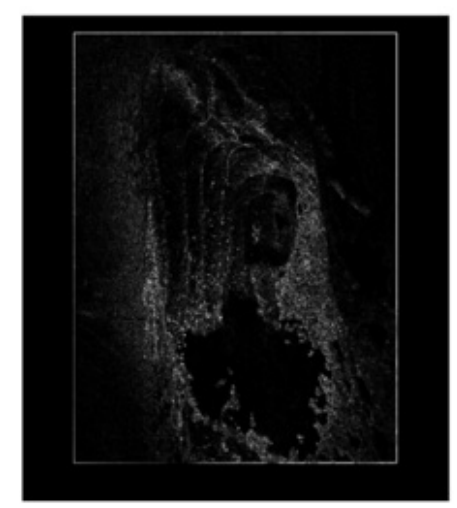

(e) Specimen of weld forming difference

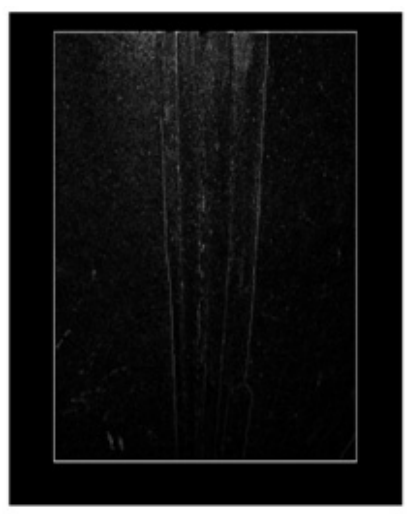

(f) Normal specimen of weld

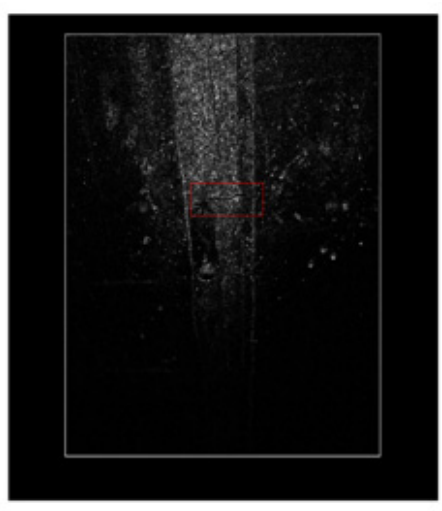

(g) Sample of cracked weld

Figure. 4 Weld sample picture

The hardware platform for this article is Windows 10 system, the processor is Intel(R) Core(TM) I7-8550U, and the installed memory is $32 \mathrm{~GB}$. In this paper, the training and testing of convolutional neural networks are based on Deep Learn Toolbox- Master in Matlab. In the training process of convolutional neural network, the learning rate, Batch, iteration times and other learning rates of the network are debuted, and the debugging of network parameters is crucial to the accuracy and efficiency of network testing.

The learning rate refers to the magnitude of updating network weights in the optimization algorithm. The learning rate can be constant, momentum-based or selfapplicable. Too large learning rate may lead to network training can never meet the requirements, too small learning rate will make network training efficiency slow, sometimes there will be a local optimal situation. Batch refers to the number of images that are input into the convoluted neural network each time. Under controllable conditions, the larger the Batch value is, the better the value will be. The larger the value is, the more accurate the descending direction of the model will be, and the 
smaller the oscillation will be; however, too much Batch will cause the convergence accuracy to fall into different local values. The number of iterations refers to the number of traverses of the data set during model training. The number of iterations will directly affect the classification accuracy of the model[5]. According to the training of convolutional neural network, the learning rate, Batch value and number of iterations were finally determined in this paper as $0.2,70$ and 6000 . The test set images were input into the trained convolutional neural network, and the accuracy of the final test results was shown in Table 1.

Table 1. Test results

\begin{tabular}{|c|c|c|c|}
\hline & Normal & $\begin{array}{l}\text { Forming } \\
\text { Difference }\end{array}$ & Cracked \\
\hline $\begin{array}{l}\text { Number of } \\
\text { tests }\end{array}$ & 200 & 100 & 100 \\
\hline $\begin{array}{l}\text { Correct } \\
\text { number }\end{array}$ & 195 & 94 & 91 \\
\hline Accuracy & $97.5 \%$ & $94 \%$ & $91 \%$ \\
\hline
\end{tabular}

Based on the test results, it can be seen that the convolutional neural network in this paper has a high recognition accuracy rate for detecting and identifying whether there are weld defects and cracks in the weld seam of the fan tower.

\section{Conclusion}

At present, the external defect detection of welding seams of fan tower tube is mainly through manual detection. The detection method based on convolutional neural network proposed in this paper is characterized by short time consuming and high robustness, and has a high detection accuracy after experimental testing. In the subsequent research, it is necessary to further improve the number and diversity of learning samples, enhance the collection quality of samples, and optimize the structure and parameters of the neural network, so as to improve the detection accuracy of the network.

\section{Acknowledgments}

Thanks to Mr. Sun's careful guidance, in the process of writing the paper, Mr. Sun helped me solved the technical problems. The work presented in this paper is supported by the Plateau Disciplines in Shanghai.

\section{References}

1. Cheng Chong, Zhang Jinqiang, Dai Jie. Visual inspection system for stainless steel welds based on convolutional neural network [J]. Industrial control computer,2019,32(05):51-52+55.

2. Shi Yangjun. Application of 3D laser scanning technology in verticality detection of fan tower [J]. Mine surveying,2019,47(02):110-115.

3. Ji Shengpeng, Yan Zhihong. Visual inspection of TIG welding seam formation based on deep learning [J/OL]. Hot working process:1-7[2020-11-07].
4. ZHAO Haiwen, ZHAO Yachuan, QI Xingyue, LI Feng. Research on Surface Defect Inspection Algorithms of Automobile Hub Based on Deep Learning $[\mathrm{J}]$. Modular machine tool and automatic machining technology,2019(11):112-115.

5. Qiao Li. Research and system design of workpiece defect detection method based on CNN [D]. Central China Normal University, 2016 